Revue internationale P.M.E.

Économie et gestion de la petite et moyenne entreprise

\title{
La diffusion des connaissances vers les PME : vers un modèle d'exploration collective
}

\section{Corine Genet}

Volume 20, numéro 1, 2007

URI : https://id.erudit.org/iderudit/1008511ar

DOI : https://doi.org/10.7202/1008511ar

Aller au sommaire du numéro

Éditeur(s)

Presses de l’Université du Québec

ISSN

0776-5436 (imprimé)

1918-9699 (numérique)

Découvrir la revue

Citer cet article

Genet, C. (2007). La diffusion des connaissances vers les PME : vers un modèle d'exploration collective. Revue internationale P.M.E., 20(1), 91-119.

https://doi.org/10.7202/1008511ar
Résumé de l'article

Si les relations entre la recherche publique et l'industrie s'établissent " naturellement " avec les grandes entreprises, il en va différemment avec les petites entreprises à faible densité de recherche. Comprendre comment les organismes de recherche publics peuvent améliorer la diffusion des connaissances vers les PME constitue le principal objectif de cet article. Notre analyse montre que deux modes distincts de production/diffusion des connaissances vers l'industrie coexistent. Le premier, basé sur la coproduction de la demande et des connaissances, se caractérise par la simultanéité du processus de production et de diffusion. Le second, basé sur l'exploration collective et la standardisation de la demande et des connaissances, dissocie la phase de production et de diffusion dans le temps mais développe une stratégie d'organisation permettant une totale connexion des deux phases. Alors que le premier mode fonctionne de manière plutôt satisfaisante pour le transfert de connaissances vers les grandes entreprises ou les PME high-tech, le second semble plus adapté aux coopérations entre la recherche publique et les PME faiblement intensives en recherche. Cet article met en perspective ces deux modèles à travers deux études de cas et en dégage quelques enseignements en termes de management des relations public/privé.
Ce document est protégé par la loi sur le droit d'auteur. L'utilisation des services d'Érudit (y compris la reproduction) est assujettie à sa politique d'utilisation que vous pouvez consulter en ligne.

https://apropos.erudit.org/fr/usagers/politique-dutilisation/ 


\title{
La diffusion des connaissances vers les PME: vers un modèle d'exploration collective
}

\author{
Corine GENET \\ Grenoble École de Management
}

\section{MOTS CLÉS}

\section{PME - Capacité d'absorption - Relations public-privé Innovation - Diffusion des connaissances}

\begin{abstract}
L'AUTEURE
CORINE GENET est enseignante-chercheure au sein de Grenoble École de management où elle enseigne la stratégie des entreprises de biotechnologie et le management de l'innovation. Elle est titulaire d'un doctorat en sciences économiques depuis 2001 et développe sa recherche autour de thèmes axés sur le management de l'innovation technologique. Adresse: Grenoble École de Management, 12, rue Pierre-Sémard, B.P. 127, Grenoble, Cédex 1, France. Téléphone: 334767062 97. Courriel : <corine.genet@grenoble-em.com>.
\end{abstract}

\section{RÉSUMÉ}

Si les relations entre la recherche publique et l'industrie s'établissent "naturellement » avec les grandes entreprises, il en va différemment avec les petites entreprises à faible densité de recherche. Comprendre comment les organismes de recherche publics peuvent améliorer la diffusion des connaissances vers les PME constitue le principal objectif de cet article.

Notre analyse montre que deux modes distincts de production/diffusion des connaissances vers l'industrie coexistent. Le premier, basé sur la coproduction de la demande et des connaissances, se caractérise par la simultanéité du processus de production et de diffusion. Le second, basé sur l'exploration collective et la standardisation de la demande et des connaissances, dissocie la phase de production et de diffusion dans le temps mais développe une stratégie d'organisation permettant une totale connexion des deux phases. Alors que le premier mode fonctionne de manière plutôt satisfaisante pour le transfert de connaissances vers les grandes entreprises ou les PME high-tech, le second semble plus adapté aux coopérations entre la recherche publique et les PME faiblement intensives en recherche. Cet article met en perspective ces deux modèles à travers deux études de cas et en dégage quelques enseignements en termes de management des relations public/privé. 


\section{ABSTRACT}

The relations between public research and SMEs are not established as "naturally" as they are with the large companies. To understand how public research institutes can improve the diffusion of knowledge to SMEs is the main objective of this article.

Our analysis shows that two organisational modes of knowledge production/ diffusion can be identified: co-production vs exploration. The first one is based on the co-production of knowledge within an interactive process of firm-university. This mode is characterised by the simultaneous process of knowledge production and diffusion. The second mode separates the stage of production from the stage of diffusion but it develops an organisational strategy to connect them. This paper argues that the co-production mode is more appropriate for the transfer of knowledge between public research institutions and high-tech SMEs or big firms. The exploration mode seems to be more adapted for cooperations between public research institutions and lowtech SMEs. This article illustrates the two modes through cases studies and gives some implication in terms of public/private relationships management.

\section{RESUMEN}

Si las relaciones entre la investigación pública y la industria se establecen de manera natural con las grandes empresas, no es lo mismo con las pequeñas empresas tradicionales. Comprender como los organismos de investigación públicos pueden mejorar sus relaciones con las PyMEs se ha convertido en un reto importante en materia de política pública de innovación. Además de la cuestión de transferencia, la mejora de la difusión de conocimientos entre los organismos de investigación públicos y las PME se plantea también la cuestión del modo de producción de conocimientos.

Nuestro análisis muestra que dos modos distintos de producción/difusión de conocimientos hacia la industria coexisten. El primero basado en la co-producción de la demanda y de los conocimientos, se caracteriza por la simultaneidad del proceso de producción y de difusión. El segundo basado sobre la exploración colectiva y la estandardización de la demanda y de los conocimientos, disocia la fase de producción y de difusión en el tiempo pero desarrolla una estrategia de organización que permite una cohesión total de las dos fases. Mientras que el primer modo funciona de manera más bien satisfactorio para la trasferencia de conocimientos hacia las grandes empresas o las PyMEs high-tech, el segundo parece más adaptado a las cooperaciones entre la investigación pública y las PME low-tech. Este artículo pone en perspectiva ese modelo de producción/difusión de conocimientos hacia las PyMEs tradicionales a la luz de un caso de cooperación entre un instituto de investigación pública y una pequeña empresa. 


\section{ZUSAMMENFASSUNG}

Die Beziehung zwischen öffentlicher Forschung und grossen Unternehmen entsteht fast automatisch; im Gegensatz zu kleinen Unternehmen mit wenigen Forschungsaktivitäten. Die grundlegende Zielsetzung des Artikels besteht darin, zu verstehen, wie die öffentlichen Forschungsorgane ihre Erkenntnisse unter den kleinen und mittleren Unternehmen besser verbreiten können.

Unsere Analyse zeigt auf, dass zwei verschiedene Modelle zur Diffusion von Forschungserkenntnissen nebeneinander bestehen. Das erste Modell basiert auf der Koproduktion von Forschungsbedarf und Erkenntnissen und ist durch die Parallelität von Erzeugung und Diffusion von Erkenntnissen charakterisiert. Das zweite Modell basiert auf der gemeinsamen Erforschung und Standardisierung der Erkenntnisse und trennt zeitlich die Phase der Produktion von jener der Diffusion, die jedoch organisatorisch absolut miteinander verbunden sind. Das erste Modell liefert zufrieden stellende Ergebnisse beim Wissenstransfer zu grossen Unternehmen oder zu kleinen und mittleren Unternehmen aus der Hightech-Branche. Während das zweite Modell angepasster erscheint für die Zusammenarbeit zwischen öffentlichen Forschungseinrichtungen und kleinen Unternehmen mit bescheidenen Forschungsaktivitäten. Der Artikel begutachtet beide Modelle anhand von zwei Fallstudien und formuliert daraus einige Erkenntnisse, die für das Beziehungsmanagement zwischen öffentlichen und privaten Institutionen herangezogen werden können.

\section{Introduction}

L'innovation dans les PME et ses liens avec la recherche scientifique est devenue l'un des thèmes phares des responsables politiques. Plusieurs concertations (Bayh-Dole Act, 1980'1 ; Livre vert sur l'innovation - Commission européenne, 1995; le rapport Chabbal, 1993; le rapport Guillaume, 1998; le Conseil européen sur la stratégie de Lisbonne, 2005) soulignent les enjeux que représente le rapprochement entre la recherche publique et les PME en matière d'innovation. Le premier argument généralement avancé en faveur des PME met en avant leur rôle dans le processus de création de la richesse et de l'emploi. D'après l'OCDE, en 2004, plus de $95 \%$ des firmes sont des PME et elles contribuent pour approximativement $60 \%$ à $70 \%$ des emplois des pays membres. En France, les petites et moyennes entreprises assurent $37 \%$ du chiffre d'affaires de l'industrie et plus de la moitié de l'emploi industriel, SESSI/DiGITIP (2003-2004). Selon les dernières enquêtes (SESSI-CIS3) effectuées entre 1998 et 2000 par le ministère de l'Économie, des Finances et de l'Industrie, 6 PME sur 10 innovent régulièrement.

1. Les termes de la Bayh-Dole Act accordent une préférence aux petites entreprises en ce qui concerne le transfert des technologies fédérales et exigent que les produits issus des transferts de ces technologies soient majoritairement fabriqués aux États-Unis. 
Différents auteurs (Pavitt, Robson et Townsend, 1987; Jaffe, 1989; Acs, Audretsch et Feldman, 1992; Audretsch et al., 2002) insistent aussi sur le caractère innovant des PME. Pour expliquer comment ces entreprises innovent, ces auteurs avancent des arguments complémentaires. À partir d'une étude multisectorielle de données américaines, Jaffe (1989) souligne la nature collective de l'innovation conduite par les PME. Lorsque Audretsch et Feldman (1996) cherchent à comprendre d'où les PME innovantes tirent les facteurs générateurs de savoir, ils parviennent à une explication très similaire. Ils montrent que les PME puisent les connaissances dont elles ont besoin pour innover dans leur environnement et, plus particulièrement, dans les universités. Leurs résultats révèlent également que les retombées issues de la recherche publique sont plus profitables aux PME qu'aux grandes entreprises. Autrement dit, les petites entreprises tirent un meilleur parti que les grandes de la recherche produite par les organismes de recherche publics (Link et Rees 1990).

Ces différentes analyses tendent donc à montrer que pour innover, les PME puisent une part des ressources dont elles ont besoin auprès d'acteurs extérieurs comme les universités, les organismes de recherche publics ou encore d'autres entreprises. Ses relations permettent notamment aux entreprises à faible capacité technologique d'acquérir les informations, les connaissances, les appuis techniques et les conseils, qui contribuent à améliorer leur niveau de connaissances scientifiques et techniques. De telles relations peuvent aussi déboucher à plus ou moins long terme sur des innovations technologiques ${ }^{2}$.

Mais, pour ce faire, les organismes de recherche publics doivent intensifier leurs liens avec les PME qui restent encore trop faibles. En effet, moins de $10 \%$ des PME innovantes auraient régulièrement recours à des laboratoires extérieurs, centres techniques, universités ou organismes de recherche (Annales des Mines, 1998, p. 96). Devant ce constat, il convient

2. Pour définir ce qu'on entend par innovation technologique, nous nous basons sur les définitions de produits et procédés innovants données par le manuel d'Oslo de l'OCDE: «un produit est considéré comme technologiquement innovant s'il donne lieu à la création d'un nouveau marché ou s'il peut se distinguer substantiellement de produits précédemment fabriqués, d'un point de vue technologique ou par des prestations rendues à l'utilisateur. Un procédé est considéré comme technologiquement innovant quand il met en œuvre de nouvelles techniques, pour la production de produits innovants, mais aussi pour la production de produits déjà existants dans la gamme de l'entreprise». Lorsque dans la communication nous faisons référence aux innovations issues de relations entre un laboratoire de recherche et une PME, nous faisons implicitement référence à ce type d'innovation. 
de se demander: quel mode de diffusion vers les PME faiblement intensives en recherche permettrait à ces dernières d'avoir accès aux connaissances produites par la recherche publique? Tel est l'objet de cet article.

Cet article appréhende le transfert comme un processus dynamique et interactif où les phases de production et de diffusion des connaissances ne sont pas des opérations parfaitement distinctes. Il montre que pour traiter la question du transfert, l'analyse de la diffusion des connaissances est indissociable de leur mode de production. Autrement dit, la façon dont sont produites les connaissances détermine leur diffusion. Cette perspective nous a incitée à mener une analyse organisationnelle des modes de production/diffusion des connaissances de deux instituts de recherche publics (l'Institut national de recherche agronomique et le Commissariat à l'énergie atomique) ${ }^{3}$ vers les PME.

La première partie propose de revenir sur les vecteurs de diffusion des résultats de la recherche publique vers les PME à faible contenu en recherche. Nous montrons que la capacité d'absorption limitée de ces entreprises ne leur permet pas d'accéder aux modes de diffusion classiquement usités par les grands groupes industriels ou les PME high-tech. Seules les relations de coopération apparaissent comme des modes de production et diffusion des connaissances pertinents pour ce type d'entreprise.

La deuxième partie présente deux modes de production/diffusion des connaissances vers les PME. L'analyse de 48 cas de coopération entre deux instituts de recherche publics (INRA et CEA) et des PME a permis de relever deux modèles alternatifs: le modèle de coproduction et le modèle d'exploration collective. L'exposé de ces deux modèles montrera que si le premier fonctionne pour les grandes entreprises et les PME high-tech, le second semble plus approprié pour la diffusion vers les PME faiblement intensives en recherche.

La troisième partie illustre les deux modèles et débouche sur une discussion mettant en exergue les éléments de fonctionnement du mode d'exploration collective.

3. De statut différent, l'INRA est un EPST alors que le CEA est un EPIC; ces instituts ont la particularité commune d'être tous deux des organismes dits à «recherche finalisée». 


\section{La diffusion des connaissances de la recherche publique vers les PME traditionnelles}

Pour diffuser leurs résultats de recherche vers les entreprises, les organismes de recherche publics disposent de plusieurs modalités: les publications, la mobilité du capital humain (Mansfield, 1995) ${ }^{4}$, la création d'entreprise, la vente et les relations formalisées ou non à travers un contrat (Siegel et al., 2001). Outre les liens de coopération, la faible capacité d'absorption des PME à faible densité de recherche ne leur permet pas d'accéder aux autres modes de diffusion des connaissances.

\subsection{Les $P M E$ à faible intensité de recherche: une capacité d'absorption limitée}

La faible capacité d'absorption des PME dépourvues de compétences de R-D en interne ne leur permet pas d'assimiler facilement des connaissances externes, même si ces dernières sont sous une forme codifiée. D'après Cohen et Levinthal (1989), la capacité d'absorption d'une entreprise correspond à sa capacité à reconnaître des savoirs externes, à les assimiler et à les exploiter : «What we call a firm's learning or absorptive capacity is its ability to identify, assimilate and exploit new knowledge. » Ils montrent que les investissements en R-D développent les capacités d'une firme à repérer, assimiler et exploiter les connaissances extérieures. Ils soulignent ainsi la double nature de la R-D. D'une part, les entreprises investissent en R-D pour générer des innovations; d'autre part, ces activités de recherche contribuent à la constitution et à l'élargissement de la capacité d'absorption de la firme. L'engagement de l'entreprise dans la production de connaissances reste donc une condition préliminaire à l'assimilation des externalités de la recherche produite par les organismes de recherche publics et les autres entreprises. De tels liens entre investissements en R-D et capacité d'absorption laissent supposer que les entreprises démunies de département de recherche interne comme la plupart des PME traditionnelles ne peuvent que difficilement assimiler et intégrer les savoirs externes. Dans le même sens, Rosenberg (1990) insiste sur les complémentarités entre les activités de recherche développées en interne par la firme et les connaissances scientifiques extérieures que la firme peut acquérir. Ces trois auteurs font donc ressortir les complémentarités entre les connaissances développées au cœur de l'entreprise et les flux externes de connaissances scientifiques et techniques. Ils considèrent la capacité d'absorption comme un produit lié des investissements en R-D, mais restent assez flous sur les

4. Mansfield (1995) montre notamment que les stages étudiants constituent un trait d'union entre les universités et les entreprises. 
éléments constitutifs de la capacité d'absorption d'une entreprise. Arora et Gambardella (1994) tentent de pallier cette insuffisance en cherchant à établir la nature de la capacité d'absorption. Pour ce faire, ils proposent de distinguer les capacités scientifiques et les capacités technologiques de l'entreprise. Les premières sont nécessaires pour l'évaluation de la pertinence des projets de coopération avec d'autres organisations. Il s'agit à la fois d'un moyen pour accroître et diversifier les connaissances disponibles dans l'entreprise et d'un processus de spécialisation scientifique. Les secondes reposent sur la faculté de la firme à utiliser la connaissance et conduisent à des innovations. Arora et Gambardella révèlent ainsi que la capacité d'absorption n'est pas seulement un produit lié des investissements en R-D, mais relève aussi d'une démarche volontaire et positive des entreprises.

Ces différents apports tendent à montrer que les PME traditionnelles, démunies de recherche interne, disposent d'une capacité d'absorption limitée. Leurs facultés à identifier, assimiler et exploiter les connaissances produites par les organismes de recherche publics sont donc assez faibles. Les vecteurs de transmission traditionnels des informations scientifiques et techniques telles que les publications savantes ou colloques restent difficilement accessibles aux PME à faible capacité de recherche. C'est pourquoi l'interaction apparaît comme un mode privilégié de diffusion des connaissances.

\subsection{L'interaction comme mode de diffusion privilégié}

La diffusion par les publications apparaît comme la modalité la plus traditionnelle et la plus «naturelle» pour des organisations de production de savoirs. Mais si les publications sont des vecteurs de divulgation des connaissances reconnus et utilisés par les grandes entreprises, les PME high-tech et les membres du monde universitaire, il n'en est pas de même pour les PME à faible capacité de recherche qui ont difficilement accès à ce type de littérature. Le manque de personnel hautement qualifié et l'écart culturel de ces entreprises avec les laboratoires publics en sont sans doute les principales causes. La mobilité du capital humain, soit à travers l'échange de personnel entre le public et le privé, soit par l'embauche de jeunes chercheurs constitue une autre modalité de diffusion du savoir vers l'industrie. Mais là encore, les moyens financiers limités des PME traditionnelles et leur faible niveau scientifique leur permettent très rarement d'établir des échanges de personnel avec les laboratoires de recherche publics ou d'employer un titulaire de doctorat, et cela, malgré l'existence d'incitations publiques prévues à cet effet. La création d'entreprises à partir de la valorisation des résultats de la recherche publique donne rarement naissance à des PME à faible capacité de recherche, les firmes ainsi créées s'inscrivent généralement dans des secteurs 
hautement intensifs en R-D. La vente, à travers la cession de licence, est, pour les organismes de recherche publics, un autre vecteur de diffusion vers le monde industriel. Mais pour être efficacement exploitée par des PME à faible contenu en recherche, encore faut-il qu'une cession de licence s'accompagne de relations de coopération en amont et en aval. De nombreux échanges et interactions entre les émetteurs et les utilisateurs sont souvent nécessaires pour que l'assimilation, l'intégration et l'exploitation d'une licence par une PME à faible capacité de recherche soit effective. Sans de tels liens avant et après la cession de licence, il y a de fortes probabilités pour que le contrat ne débouche sur aucune innovation ou amélioration significative de la base de connaissances scientifiques et techniques de l'entreprise. Comparativement à l'ensemble des modalités de diffusion que nous venons d'exposer, les relations de coopération apparaissent comme un mode de diffusion plus adapté à la faible capacité d'absorption qui caractérise les PME traditionnelles. Mangematin et Nesta (1999) corroborent statistiquement ce point.

Grâce notamment au processus d'apprentissage cumulatif qu'elles déclenchent, les relations de coopération permettent de résorber l'écart culturel, scientifique et technique qui existe entre les chercheurs des laboratoires de recherche publics et les membres de ces PME. Les objectifs de la recherche, la demande des entreprises et le niveau de connaissances des acteurs évoluent au cours des interactions. Ce processus interactif et constructiviste permet aux acteurs de la coopération d'apprendre, d'évoluer et de modifier ainsi leur base de connaissances et leur trajectoire. L'émergence d'un tel processus d'apprentissage est soumise à plusieurs conditions organisationnelles. Par exemple, il est essentiel que le mode de production des connaissances soit étroitement connecté aux impératifs de diffusion vers les PME. On ne peut pas raisonner et construire un mode de diffusion vers les PME indépendamment du mode de production des connaissances. Si le problème de l'amélioration des relations entre la recherche publique et les PME faiblement intensives en recherche pose la question du transfert, elle pose aussi en amont celle du mode de production, telle est notre hypothèse de départ. Cet angle d'approche nous conduit à mener une analyse des relations de coopération en tant que mode de production et diffusion des connaissances vers les PME.

\section{Modèle d'exploration collective versus modèle de coproduction}

L'analyse de cas de coopération entre deux instituts de recherche publics et des PME fait apparaître l'existence de deux modes de production/diffusion des connaissances. Alors que le premier se base sur la coproduction de la 
demande et des connaissances (2.2), le second s'appuie sur leur standardisation à travers un processus d'exploration collective (2.3). Ces deux modes se déclinent en fonction de quatre principaux critères: les connaissances échangées, l'output de la relation, le réseau de partenaires mobilisé et le degré d'indépendance entre les acteurs du processus ( $c f$. tableau 1$)$.

\section{TABleau 1}

\section{Mode basé sur la coproduction/Mode basé sur l'exploration collective}

\begin{tabular}{|c|c|c|}
\hline & $\begin{array}{l}\text { Mode de diffusion basé } \\
\text { sur la coproduction }\end{array}$ & $\begin{array}{l}\text { Mode de diffusion basé sur } \\
\text { l'exploration collective }\end{array}$ \\
\hline $\begin{array}{l}\text { Type de } \\
\text { connaissances } \\
\text { échangées }\end{array}$ & Des connaissances tacites. & Des connaissances codifiées. \\
\hline $\begin{array}{l}\text { Output de } \\
\text { la relation }\end{array}$ & $\begin{array}{l}\text { Des connaissances à usage } \\
\text { non collectif, difficilement } \\
\text { transférables à d'autres } \\
\text { entreprises. } \\
\text { Réponse idiosyncratique. }\end{array}$ & $\begin{array}{l}\text { Des connaissances à usage } \\
\text { collectif et transférables à un } \\
\text { réseau d'entreprises stabilisé. } \\
\text { Réponse standardisée. }\end{array}$ \\
\hline $\begin{array}{l}\text { Réseau de } \\
\text { partenaires } \\
\text { mobilisé }\end{array}$ & $\begin{array}{l}\text { Le réseau est construit et } \\
\text { soutenu par des relations } \\
\text { humaines, il est plus } \\
\text { ou moins stable. }\end{array}$ & $\begin{array}{l}\text { Le réseau est stable, il est soutenu } \\
\text { par des dispositifs organisation- } \\
\text { nels. }\end{array}$ \\
\hline $\begin{array}{l}\text { Degré } \\
\text { d'indépendance } \\
\text { entre les acteurs }\end{array}$ & Faible indépendance. & Forte indépendance. \\
\hline
\end{tabular}

Après une brève présentation de notre méthodologie de recueil de cas, nous exposerons successivement les deux modes de production/diffusion des connaissances que nous avons relevés et modélisés.

\subsection{Une méthodologie basée sur l'étude de cas de coopération}

Notre approche méthodologique repose sur l'étude de 48 cas de coopération entre 20 laboratoires (10 CEA, 10 INRA) et des PME à faible densité de recherche. L'analyse de ces cas de relations a apporté une meilleure compréhension des mécanismes de production et de diffusion des connaissances recherche/PME. C'est à partir de cette analyse que les deux modèles conceptuels que nous proposons ont été conçus.

Les études de cas ont été construites à partir d'entretiens en tête à tête avec les membres des laboratoires INRA et CEA. Les cas ont été directement sélectionnés par les acteurs. La personne interrogée a donné les caractéristiques générales du cas avant de répondre à une série de questions 
concernant six thèmes généraux (l'initiation de la relation, la stratégie des acteurs, la relation au quotidien, la fréquence et la nature des interactions, le comportement des partenaires pendant la relation et les retombées de la relation).

Pour mieux expliciter les deux modes de production/diffusion des connaissances relevés, 2 des 48 études de cas recueillies sont ensuite exposées dans le détail.

\subsection{Le mode de diffusion basé sur la coproduction de la demande et des connaissances}

Dans ce modèle, la production et la diffusion des connaissances sont indissociables; elles s'opèrent simultanément au cours de la relation. Par conséquent, la demande comme les connaissances ne préexistent pas, elles sont élaborées collectivement et diffusées au cours des interactions entre les chercheurs et les industriels. L'émergence d'une demande industrielle stable et des connaissances repose sur des relations de confiance étroites entre les acteurs et des échanges fréquents. C'est au cours de la coopération que les acteurs vont saisir ce qu'ils sont en mesure d'exiger de l'autre et ce qu'ils sont capables de générer en commun. Dans ce mode, la demande et les connaissances sont donc le résultat d'un processus d'apprentissage interactif et dynamique entre les partenaires: elles n'existent pas ex-ante. Sur ce point, le mode basé sur la coproduction se rapproche du modèle de coconstruction élaboré par le Centre de sociologie de l'innovation. Pour synthétiser les résultats d'une étude sur le développement et le fonctionnement des innovations à l'INRA, le CSI a mis en évidence l'existence de deux «idéaux types »: le modèle de coconstruction et le modèle de délégation ${ }^{5}$. D'après les auteurs, dans le premier modèle, l'offre et la demande ne sont pas dissociées dans le temps, mais se construisent au cours du projet commun. La production et la diffusion des connaissances sont donc perçues comme un seul et même processus. Dans ce sens, on peut dire que le mode basé sur la coproduction est proche du modèle de coconstruction du CSI. Il se rapproche également du modèle de

5. Trois principaux critères ont été utilisés pour différencier les deux modèles: la définition du marché que se donnent les acteurs, les relations qu'ils établissent avec leurs partenaires et les formes de contrats qu'ils mettent en place. Basés sur une vision très interactive du processus d'innovation, les principes du modèle de coconstruction se déclinent selon trois critères: la coconstruction du marché, la structuration du milieu et l'antériorité des relations sur les contrats. Davantage bâti sur une conception linéaire du fonctionnement et du développement des projets d'innovation, le modèle de délégation présente trois caractéristiques: le marché est représenté par le partenaire industriel, les partenariats sont déjà constitués et les contrats sont antérieurs à la constitution de relations communes. 
«conception et réalisation commune» décrit par Mangematin où les PME parviennent à innover grâce à une recherche menée en commun avec leurs partenaires (Mangematin, 1997). C'est aussi le modèle qui se rapproche le plus du cas généralement analysé par les économistes du changement technique.

Outre la simultanéité du processus de production et de diffusion, plusieurs éléments caractérisent ce mode de diffusion. Tout d'abord, la forme tacite des connaissances échangées et la nature localisée des connaissances produites. Enfin, le mode de construction des liens entre partenaires dépend exclusivement des relations interpersonnelles des individus. Le bref exposé de chacune de ces caractéristiques va permettre de mieux définir ce mode de diffusion.

\section{Des connaissances échangées principalement tacites}

Dans ce processus, les connaissances échangées entre chercheurs et industriels sont essentiellement de nature tacite (Polanyi, 1962). Incorporées dans les personnes (Callon, 1997), elles ne peuvent, dans un premier temps, faire l'objet d'une quelconque codification. La codification demeure un processus ultérieur, donc subordonné à l'existence préalable du savoir (Saviotti, 1994). Dans un processus de coproduction, les connaissances se construisent au cours des interactions entre des individus (Senker, 1995). Ainsi produites et diffusées, les connaissances ne peuvent donc pas faire l'objet d'une codification immédiate.

\section{Une réponse idiosyncratique et un fort degré de dépendance entre les acteurs}

La production et la diffusion des savoirs dans ce processus résultent de fortes interactions entre les scientifiques et les industriels. La demande et les connaissances produites sont intimement liées aux objectifs, aux besoins, aux contraintes et à la base de connaissances de l'ensemble des partenaires. Les résultats de la relation sont dépendants du contexte dans lequel ils ont été créés. C'est au cours des échanges qu'un projet stable et précis émerge et qu'une solution aux problèmes émis par l'industriel se construit. Les différentes tâches effectuées lors de la coopération sont étroitement connectées entre elles, de nombreux allers-retours entre les acteurs sont nécessaires. L'accomplissement d'une tâche dépend étroitement des connaissances, conseils et savoir-faire des chercheurs du laboratoire et des industriels. Seuls une mise en commun des savoirs et des compétences et un travail collectif permettent à la relation d'aboutir à la production et la 
diffusion de l'innovation. La production de connaissances et leur application en contexte ne sont plus des processus mutuellement exclusifs et séparés mais une démarche d'apprentissage qui réunit des «investigateurs scientifiques» et des «investigateurs professionnels» - selon le terme employé par Argyris et Schön (2002). Il en résulte une forte dépendance mutuelle entre les acteurs.

\section{Un réseau soutenu par des relations personnelles souvent informelles}

Les liens qui unissent les partenaires du processus de coproduction/diffusion de l'innovation découlent, le plus souvent, de relations antérieures informelles (relations personnelles, correspondance électronique ou téléphonique, conversations lors de colloques ou salons, etc.) ou formalisés (contrat d'expertise, de consultance, de prestation de services ou de collaboration de recherche). Ces interactions entre chercheurs et industriels constituent un cadre d'apprentissage dynamique. C'est au cours de la coopération que chercheurs et industriels vont comprendre ce qu'ils sont en mesure d'exiger de l'autre et ce qu'ils sont capables de générer. Les maintes réunions, négociations, conversations, tests qui ponctuent le processus de coopération vont progressivement permettre à la demande industrielle de se stabiliser et aux nouvelles connaissances d'émerger.

\subsection{Le mode de diffusion basé sur l'exploration collective}

Basé sur la standardisation de la demande industrielle et des connaissances diffusées, ce mode de diffusion se distingue nettement du précédent. Dans ce mode, la production et la diffusion des connaissances sont dissociées dans le temps et ne résultent pas d'un seul et même processus. Distinctes dans le temps, ces deux phases se différencient également en fonction des acteurs qu'elles mobilisent. La production des savoirs est assurée par le laboratoire et son réseau de partenaires scientifiques en amont, alors que la diffusion s'appuie sur les différents dispositifs du laboratoire et son réseau de partenaires industriels en aval. Les deux phases restent toutefois fortement connectées dans la mesure où ce sont les besoins émis par les industriels qui définissent les thématiques de la recherche collective. Les connaissances ainsi produites sont à usage collectif, standardisées et transférables par l'intermédiaire de différents dispositifs (rapports techniques, réunions, manuels techniques, bulletins d'information périodiques, contrats bilatéraux, plates-formes industrielles, halles technologiques, etc.) communs à l'ensemble des partenaires du réseau. Dans ce modèle, la stratégie de construction du mode de production des connaissances est élaborée et organisée pour la diffusion. 
On peut rapprocher ce modèle de la logique relationnelle de «club» mise en évidence par Joly et Mangematin dans un article de 1996. À partir d'une étude sur les contrats industriels de différents laboratoires de l'INRA, les auteurs montrent qu'il existe trois logiques relationnelles différentes: une logique de club ou de proximité, une logique de relation symbiotique et une logique de relation marchande. Dans la première, les industriels se regroupent en «club» pour compenser leur faible capacité d'absorption et leurs moyens de financement limités. Ce regroupement est d'autant plus opportun que l'objectif des recherches est bien souvent la production d'objets d'intérêt collectif. En ce sens, cette logique relationnelle est proche de notre modèle basé sur l'exploration collective, alors que la logique de relation symbiotique correspond plus à notre modèle basé sur la coproduction.

Pour fonctionner, ce modèle exige une configuration organisationnelle particulière. Plusieurs dispositifs organisationnels tels que le réseau de partenaires industriels, la cellule de diffusion technologique interne, un centre de ressources et de transfert, les plates-formes industrielles ou halles de technologies et le processus de recherche collective soutiennent ce modèle. Outre cette caractéristique, d'autres éléments sont propres à ce mode de diffusion.

\section{Des connaissances échangées essentiellement codifiées}

Contrairement au modèle précédent, les connaissances échangées entre les acteurs de la coopération présentent un plus fort degré de codification. Issues de programmes de recherche collective amont, les connaissances préexistent à la relation; elles n'émergent pas dans la coopération même si cette dernière contribue souvent à les enrichir. La distinction traditionnelle entre connaissances tacites et connaissances codifiées doit toutefois être saisie avec circonspection. Sur ce point, Polanyi (1962) précise que le processus de codification des connaissances reste souvent partiel, car il paraît difficile de retranscrire l'intégralité d'une connaissance. Dans ce sens, Saviotti (1994) affirme qu'il serait préférable de parler de degré de codification dont les formes tacites et codifiées des connaissances seraient les deux points extrêmes. Cette remarque s'applique tout à fait à notre modèle, où les connaissances ne sont jamais complètement codifiées.

\section{Une réponse standardisée et un fort degré d'indépendance entre les acteurs}

Dans le mode de diffusion basé sur l'exploration collective, les connaissances, produites en amont par un processus de recherche collective, peuvent être 
transférées à toutes les entreprises souhaitant les exploiter; on peut à ce titre les qualifier de génériques. Les entreprises réceptrices sont à l'origine des thèmes développés par les programmes de recherche collectifs. En effet, c'est sur la base d'un référendum auprès des industriels appartenant au réseau du laboratoire que les thématiques de recherche sont construites. Issus d'un continuum de négociation entre les chercheurs et les industriels, ces programmes de recherche, financés par des fonds publics et réalisés en partenariat avec d'autres laboratoires, produisent des connaissances génériques. Toutes les entreprises membres du réseau du laboratoire peuvent ensuite bénéficier des résultats de cette recherche collective. Ainsi, la réponse apportée aux industriels peut être qualifiée de standardisée, car elle n'est pas propre au problème d'une seule entreprise mais peut s'adapter aux besoins d'un ensemble d'entreprises. Le degré de dépendance qui lie les producteurs et les utilisateurs des savoirs est donc beaucoup plus faible que dans le cas du mode de coproduction. Sans avoir participé activement à la production, une entreprise reste néanmoins capable d'exploiter et d'utiliser les connaissances engendrées par les laboratoires.

\section{Un réseau soutenu par des dispositifs organisationnels}

Le réseau sur lequel s'appuie la diffusion des connaissances dans le mode basé sur la standardisation s'articule autour d'un dispositif organisationnel formalisé qui peut prendre la forme d'un club, d'une association ou d'un syndicat de professionnels.

Ce réseau regroupe un ensemble d'industriels connecté au domaine scientifique et technologique du laboratoire. Ce regroupement d'industriels et/ou de professionnels a comme objectif commun de bénéficier des connaissances élaborées par le laboratoire partenaire. Ces connaissances résultent principalement d'une recherche collective élaborée en amont par un collectif d'acteurs articulé autour du laboratoire et de ses partenaires scientifiques. Les thématiques génériques développées par ces programmes de recherche sont définies en fonction des besoins industriels. Pour ce faire, de fréquentes discussions entre chercheurs et industriels sont nécessaires. Ces interactions peuvent prendre la forme de réunions, d'enquêtes ou de simples conversations lors d'essais réalisés sur une plate-forme ou dans une halle technologique. Ces lieux de rencontre, qui permettent aux chercheurs et aux industriels de discuter autour d'un dispositif expérimental, peuvent agir comme des médiateurs, des filtres. Ces contacts en personne transforment peu à peu des questions imprécises en des thématiques de recherche collectives. C'est ainsi que de tels dispositifs contribuent à traduire la demande industrielle et à orienter les thèmes de recherche. Mais pour qu'une telle standardisation de la 
demande industrielle soit possible, il est nécessaire que le réseau d'entreprises partenaires soit très stable. Ensuite, différents dispositifs tels que des rapports techniques, des réunions, des manuels techniques, des bulletins d'informations, des formations, des essais réalisés dans les halles technologiques ou sur les plates-formes ou encore des contrats bilatéraux confidentiels permettent de diffuser ces connaissances vers les membres du réseau. L'élaboration et la mise en place de l'ensemble de ces dispositifs de «transmission» exige que le laboratoire soit capable d'effectuer un lourd travail de "traduction et d'adaptation ». Ce processus de «traduction et d'adaptation» donne lieu à «un va-et-vient systématique entre la conceptualisation, l'expérimentation, l'intervention et à nouveau la conceptualisation » selon les termes employés par Julien (2004).

La présentation détaillée du modèle basé sur la coproduction et du modèle basé sur l'exploration collective montre que la diffusion des connaissances vers les PME peut prendre deux formes alternatives. Alors que le premier fonctionne assez bien pour le transfert de connaissances vers les grandes entreprises ou les PME high-tech, le second semble plus adapté aux coopérations entre la recherche publique et les PME à faible intensité de recherche. Toutefois, sur les 48 cas de relations étudiés entre un laboratoire de recherche et une PME, seul un tiers des coopérations (16 sur 48) ont adopté le modèle d'exploration collective. Pour mieux appréhender les caractéristiques de fonctionnement de ces deux modes, nous proposons de les illustrer par deux études de cas.

\section{Les modes de diffusion en action: deux cas d'illustration}

Extraits des 48 études de cas de coopération recherche-PME collectés auprès de laboratoires CEA ou INRA, les cas de coopération qui suivent illustrent le fonctionnement des deux modes de diffusion présentés précédemment. Une mise en perspective des autres cas étudiés permettra ensuite de souligner les conditions organisationnelles nécessaires à l'adoption d'un modèle d'exploration collective.

\subsection{Un cas d'illustration du mode de diffusion basé sur la coproduction}

Avant d'expliciter dans le détail l'histoire de la coopération entre la station de botanique et pathologie végétale de l'INRA et la $\mathrm{PME}$ «P», un préambule sur les antécédents du projet sur «la décontamination des palmiers dattiers » s'impose. 


\subsubsection{Préambule}

La station de botanique et pathologie végétale de l'INRA basée sur le centre d'Antibes existe depuis 1965. Ses principaux axes de recherche concernent l'étude de l'expression du génome in vitro et l'étude des bases de la régénération des plantes. L'application, comme la diffusion des connaissances vers la profession, reste l'un des objectifs de la station. Dans ce domaine, l'action du laboratoire porte principalement sur la décontamination des plantes et la production de plants sains. Ainsi, lorsque les palmeraies du Maroc commencent à être décimées par une bactérie, la station s'associe à Total et au CNRS pour chercher des solutions capables d'endiguer cette contamination. L'objet est d'identifier des individus tolérants afin de mettre en place un programme de sélection, suivi d'un programme de mise en culture et de multiplication in vitro. Pour ce contrat, Total met à disposition un laboratoire de recherche à Porquerolles. Mais très vite, le groupe pétrolier décide de suspendre son financement et de fermer son laboratoire. Devant ce brutal désengagement, la direction de l'INRA choisit en 1991 de poursuivre ces recherches et de reprendre le laboratoire de Porquerolles. Pour ce faire, la station bénéficie d'un financement régional.

\subsubsection{De l'initiative d'une PME à l'intervention de la fonction d'interface}

Intéressée par ces travaux, une PME implantée en Touraine contacte en 1994 la station de botanique et pathologie végétale. Principal fournisseur des jardins royaux du Maroc, cette petite entreprise productrice de palmiers dattiers souhaite s'associer au laboratoire. Une fois le dialogue établi entre le directeur de la PME et Monsieur «B », l'ingénieur d'étude responsable de cet axe de recherche à l'INRA, un cahier des charges est rédigé. La réalisation conjointe de ce document a demandé plusieurs entrevues et discussions entre Monsieur «B» et le p.-d. g. de la PME. Les lignes du projet dessinées, le laboratoire choisit de faire appel à la fonction d'interface de son institut pour régler l'ensemble des démarches administratives propres à la contractualisation. Monsieur « $\mathrm{B}$ » explique que lorsque la communication avec l'entreprise est établie, il ne souhaite généralement pas avoir en charge la phase de contractualisation.

Entre la $\mathrm{PME} \ll \mathrm{P} »$ et la station de botanique et pathologie végétale, un contrat de collaboration de recherche sur les problèmes de contamination par bactéries des palmiers dattiers de deux ans a donc été conclu et signé en 1995. Pour l'entreprise, les enjeux de cette coopération sont énormes d'autant plus que l'introduction d'un procédé de multiplication in vitro lui permettrait de gagner beaucoup de temps pour la production des palmiers. Pour le laboratoire, l'intérêt est tout aussi important, car cette relation devrait l'amener 
à poursuivre cet axe de recherche et à prendre contact avec les régions d'Afrique du Nord pour leur proposer un protocole capable d'endiguer la contamination des palmeraies. Pour la station de botanique et pathologie végétale, cette coopération pourrait donc se traduire par de nouvelles possibilités d'application et l'ouverture vers un nouveau réseau de partenaires potentiels.

\subsubsection{Des interactions quotidiennes comme vecteurs d'échange de connaissances tacites}

Dès le début de la coopération, la société « $\mathrm{P}$ » place des ingénieurs de recherche-développement au sein du laboratoire expérimental de l'INRA à Porquerolles. L'un de ces ingénieurs, Mademoiselle « $\mathrm{C}$ », a été employée spécialement par la PME pour mener à bien cette collaboration. Le choix du candidat ne s'est pas fait au hasard. En effet, la jeune titulaire d'un doctorat que l'entreprise a recrutée lui avait été recommandée par la station qui avait accueilli la jeune femme lors de la réalisation de sa thèse. Pour sa part, la station a choisi de placer un technicien à Porquerolles, alors que Monsieur «B $\mathrm{B}$ ) assure le pilotage de la coopération à Antibes. Bien que basé à Antibes, Monsieur «B» n'hésite pas à effectuer de très nombreux déplacements à Porquerolles pour les nombreuses expérimentations in situ, les réunions et de fréquents tête-à-tête. Des échanges réguliers par télécopieur, courrier ou messagerie électronique s'ajoutent aux rencontres physiques qui ont lieu soit dans le laboratoire de Porquerolles, soit à la station d'Antibes, soit encore dans la PME à Tours.

L'essentiel du travail d'expérimentation est réalisé dans la palmeraie du laboratoire de Porquerolles, alors que l'activité de production est assurée à Tours par la PME. La présence au sein du même laboratoire des membres de la station INRA et de la PME permet un dialogue permanent et une division du travail très souple. Ainsi, une équipe travaille sur des milieux de cultures spécifiques et simultanément l'autre équipe étudie d'autres milieux de cultures, ce qui permet ensuite d'avoir suffisamment d'éléments pour réaliser une comparaison. Une telle proximité ajoutée à de nombreuses interactions formelles et informelles entre l'ensemble des participants ont été des vecteurs privilégiés pour l'échange d'informations et de connaissances. De formes essentiellement tacites, les connaissances échangées lors des expérimentations communes à Porquerolles ou lors de conversations ont permis à la PME d'intégrer de nouvelles méthodes de culture. Comme l'entreprise, la station a aussi acquis de nouvelles connaissances au cours de cette relation de coopération. En effet, si le laboratoire INRA connaît bien les techniques de 
multiplication in vitro qu'il pratique depuis 30 ans, il a appris à appliquer ces techniques sur le palmier dattier qui, compte tenu des difficultés, représente une variété rarement cultivée in vitro.

\subsubsection{L'output de la relation: la mise au point de deux procédés}

À l'issue du contrat d'une durée initiale de deux ans, prolongé par un avenant, deux procédés de mise en culture et de multiplication de palmiers dattiers sans manifestation de bactéries ont été mis au point. De tels résultats ont permis à la PME d'améliorer sa production et de mettre sur le marché une nouvelle variété de palmiers dattiers sains. Les bénéfices de la coopération ont été tout aussi importants pour la station de botanique et pathologie végétale. En effet, cette relation a permis l'introduction d'une nouvelle thématique de recherche (l'amélioration de la variété palmier dattier), d'un nouveau secteur d'application (les palmeraies d'Afrique du Nord), de deux nouvelles méthodologies (procédés de mise en culture) et de nouveaux équipements et instruments. Cette relation a aussi été, pour le laboratoire, l'occasion d'établir de nouveaux contacts avec d'autres industriels et d'autres laboratoires publics tunisiens et marocains. De telles ouvertures vont permettre à la station de former un nouveau réseau articulé autour des pays d'Afrique du Nord qui peuvent être intéressés par les travaux réalisés sur le palmier dattier.

Le transfert des résultats de cette recherche vers d'autres partenaires constitue désormais l'un des objectifs de la station INRA. Mais, compte tenu des clauses de confidentialité et du caractère localisé des connaissances coproduites lors de la coopération, une plus large diffusion vers d'autres partenaires paraît difficilement réalisable dans l'immédiat. Du temps et d'autres coopérations seront nécessaires pour poursuivre la construction du nouveau réseau de partenaires et adapter les résultats issus de la relation avec la $\mathrm{PME}$ «P » à d'autres partenaires, d'autres contextes, d'autres besoins et d'autres compétences et capacités.

\subsection{Un cas d'illustration du mode de diffusion basé sur l'exploration collective}

Afin de mieux comprendre l'ensemble des éléments qui caractérisent le fonctionnement du mode de diffusion basé sur l'exploration collective dans ce cas d'étude, il convient, avant de relater la coopération telle qu'elle s'est déroulée, de faire le point sur l'organisation du laboratoire impliqué. 


\subsubsection{Le GRETh: un laboratoire particulier}

Le Groupement pour la recherche sur les échangeurs thermiques (GRETh) a été créé en 1984 par l'Agence de l'environnement et de la maîtrise de l'énergie (ADEME) et le CEA. La compétence centrale de ce laboratoire porte sur la thermique industrielle. Depuis 1997, le GRETh est un Centre de ressource technologique (CRT). En tant que CRT, ce laboratoire a pour mission légitime de diffuser ses résultats vers l'industrie et, plus particulièrement, vers les PME-PMI. De ce fait, une partie non négligeable de l'activité des membres du GRETh est consacrée à la collaboration avec l'industrie. La prospection, le conseil, l'expertise sont intégrés comme des formes d'activités légitimes au même titre que les activités de recherche. Pour remplir sa mission auprès des industriels, le GRETh s'est doté d'une structure organisationnelle particulière.

Outre ses deux laboratoires de recherche spécialisés (L3EI, Laboratoire d'étude sur l'efficacité énergétique dans l'industrie; L3EBT, Laboratoire d'étude sur l'efficacité énergétique dans le bâtiment et les transports), le GRETh dispose d'une cellule de diffusion technologique (CDT) comprenant principalement une personne, trois plates-formes expérimentales et un club d'industriels (l'association GRETh). La cellule de diffusion et les plates-formes sont des dispositifs transversaux aux deux laboratoires de recherche qui sont en interaction constante avec l'association GRETh. Une telle structure donne les moyens aux membres du laboratoire de pratiquer une activité de diffusion vers l'industrie.

En plus de sa structure particulière, le GRETh se caractérise par son mode d'organisation. Ce CRT s'organise autour de trois pôles d'activité: le pôle recherche collective (programmes de recherche fondamentale et de diffusion), le pôle de recherche finalisée et les prestations. Les programmes de recherche fondamentale, financés par des fonds publics (CEA, ADEME, CNRS, EDF et Union européenne) et le programme de diffusion, principalement financé par la cotisation des industriels membres du club, comptent pour environ $40 \%$ de l'activité du laboratoire. La diffusion des connaissances vers les membres du club est prise en charge par la cellule de diffusion (CDT) et l'association GRETh à travers des dispositifs tels que les rapports techniques, le manuel technique, les bulletins périodiques, les réunions, etc. Le pôle de recherche finalisée est cofinancé à $50 \%$ par le CEA et à $50 \%$ par les partenaires industriels et/ou publics (CEE, Agences de programme) du GRETh et représente $30 \%$ de son activité. Les prestations et autres contrats sont uniquement financés par l'industrie et constituent $30 \%$ de l'activité du laboratoire. 


\subsubsection{Une réunion thématique comme élément déclencheur de la coopération}

L'évolution des réglementations nationales et européennes sur les rejets d'effluents gazeux favorise le développement de technologies plus performantes de destruction ou de captation des composés organiques volatils. Depuis quelques années, le GRETh développe plusieurs axes de recherche collective sur ce sujet. Pour évaluer les premiers résultats de cette recherche et continuer à orienter les thématiques de recherche en fonction des besoins industriels, le laboratoire organise une réunion thématique sur le traitement des effluents gazeux. Toutes les entreprises reconnues dans ce secteur sont invitées. Parmi l'ensemble de ces industriels se trouve la $\mathrm{PME}$ «S , société d'ingénierie lyonnaise spécialisée dans le domaine. Lors de cette réunion, le directeur technique de la $\mathrm{PME}$ « $\mathrm{S}$ » sollicite Monsieur « $\mathrm{M} »$, l'un des chargés d'affaires du GRETh. Après discussion entre les deux individus, une rencontre chez l'industriel est convenue. Cette visite permet à l'industriel d'exposer ses problèmes et ses questions.

Cette petite société d'ingénierie a mis au point, pour un important groupe pharmaceutique, une installation pour le traitement d'effluents gazeux. Il s'agit, pour cette entreprise, de la première installation de ce type qu'elle conçoit. Après livraison, le client souhaite une évaluation des performances de l'appareillage. Or la $\mathrm{PME} \ll \mathrm{S}$ » ne possède pas en interne les moyens nécessaires pour analyser et mesurer les performances de cette nouvelle installation. C'est pour répondre aux exigences de ce client que l'entreprise sollicite l'expertise du GRETh dans ce domaine.

Après cette première visite, le chargé d'affaires du GRETh, Monsieur «M», possède suffisamment d'éléments sur la demande de l'entreprise pour rédiger une proposition technique et financière qu'il soumet à la PME dès septembre 1996. Après accord sur les termes de la coopération avec l'industriel, Monsieur « $\mathrm{M} »$ rédige un contrat de prestation de services d'une durée de six mois et d'un montant de 200000 francs qui est signé par le directeur de la PME et le directeur du GRETh en décembre de la même année.

\subsubsection{Des interactions articulées autour de quatre types d'acteurs}

Pour l'industriel, l'intérêt de cette coopération est clair: elle doit lui permettre de répondre à la demande de son client et d'améliorer sa première installation industrielle qui doit absolument respecter les nouvelles normes en matière de rejets d'effluents gazeux. Pour le laboratoire, cette relation répond aux 
objectifs de «service public» que lui a attribués sa direction et représente l'occasion de se positionner sur un nouveau domaine d'application: la qualité de l'air et le traitement des effluents.

La division du travail entre les deux parties est simple. L'entreprise se contente d'apporter le soutien logistique, alors que le laboratoire effectue l'ensemble des différentes tâches nécessaires à l'expertise (mesures, analyses, etc.). Pour mener à bien cette expertise, l'échange de données codifiées sur les plans de l'échangeur thermique et de l'installation et sur le planning de production du client utilisateur a été capital. L'échange d'informations entre les membres du GRETh et le chargé d'affaires de la PME s'est principalement effectué lors de rencontres dans l'entreprise et des réunions organisées tous les deux mois par le GRETh. Ces réunions de travail avaient lieu soit dans la $\mathrm{PME}$ «S», soit chez le client exploitant qui a parfois souhaité participer.

L'ensemble des interactions qui ont rythmé cette coopération n'a pas seulement impliqué certains membres du GRETh et de la PME «S », deux autres partenaires ont été partie prenante. Tout d'abord le client, exploitant de l'installation industrielle qui, en tant que demandeur, a toujours montré beaucoup d'intérêt à l'expertise. Outre les informations qu'il a régulièrement fournies, plusieurs mesures et essais ont été réalisés par le GRETh sur son site.

En plus du groupe pharmaceutique exploitant, une autre entreprise a indirectement participé à cette coopération: le sous-traitant de la PME «S », fabricant de l'échangeur thermique. Cette troisième entreprise n'était pas inconnue du laboratoire puisqu'elle est l'un des adhérents du club du GRETh. Mais, plus que quatre groupes d'acteurs, il faut également relever toute l'importance d'un objet: l'installation, qui a été le pivot de la coopération entre tous les participants. En effet, c'est autour de cet objet que les relations entre le GRETh et la PME, la PME et le sous-traitant, et le groupe pharmaceutique et la PME se sont articulées.

\subsubsection{Un nouvel adhérent pour le club du GRETh}

Cette première coopération a été source de gains pour la $\mathrm{PME}$ « $\mathrm{S}$ », car elle a pu ainsi trouver les points critiques de son procédé, mettre en place des pistes d'amélioration et surtout gagner une certaine crédibilité auprès de son premier client dans le domaine. Son adhésion au club d'industriels du GRETh lui a permis de bénéficier gratuitement d'une seconde expertise et d'un suivi pour la nouvelle installation qu'elle a ensuite conçue. 
Cette prestation de services a permis au GRETh de pénétrer un nouveau secteur d'application, à savoir le traitement des effluents gazeux, d'appliquer et de tester une partie des connaissances élaborées en amont lors des programmes de recherche collective. En termes d'image, cette relation a aussi été fructueuse pour le GRETh (articles dans la presse professionnelle et la réalisation de différentes communications lors de séminaires).

D'un point de vue relationnel, cette coopération a permis au GRETh de pénétrer un nouveau réseau d'acteurs articulé autour du traitement des effluents gazeux. Cette ouverture s'est notamment traduite par l'établissement d'un nouveau contrat d'étude sur le sujet avec le sous-traitant, sur une nouvelle expertise gratuite pour la $\mathrm{PME}$ «S» et sur l'élaboration d'un nouveau programme de R-D. Plusieurs industriels, dont la $\mathrm{PME}$ « $\mathrm{S}$ », sont impliqués dans cet axe de recherche collective. Ce nouveau programme a débouché sur la réalisation d'un nouveau moyen d'essai (boucle d'essai CLARA financée par la région, l'ADEME et le CEA), le dépôt de deux brevets et la création d'une entreprise.

\subsection{Le modèle d'exploration collective, un modèle adapté à la diffusion des connaissances vers les PME?}

Le cas relatant «le traitement d'effluents gazeux» par le GRETh et une PME lyonnaise et le cas de «la décontamination du palmier dattier» à l'INRA ont mis en valeur les différentes caractéristiques qui distinguent le mode de diffusion basé sur l'exploration collective du mode de diffusion sur la coproduction. Le tableau 2 synthétise les éléments clés du fonctionnement des modes de diffusion pour les deux cas d'illustration présentés.

La présentation détaillée des deux cas permet tout d'abord de souligner la différence des formes d'interactions entre acteurs et la nature des connaissances échangées qui varie d'un mode de diffusion à l'autre. Si entre le GRETh et la PME lyonnaise les principaux échanges sont très formalisés et articulés autour de réunions ou d'objets (installations), il n'en est pas de même pour le laboratoire de l'INRA et la PME où les interactions sont beaucoup plus fréquentes et informelles. Les rencontres de face à face, la réalisation d'expériences en commun dans le laboratoire expérimental de Porquerolles ont largement facilité l'échange de connaissances tacites entre les deux entités. 


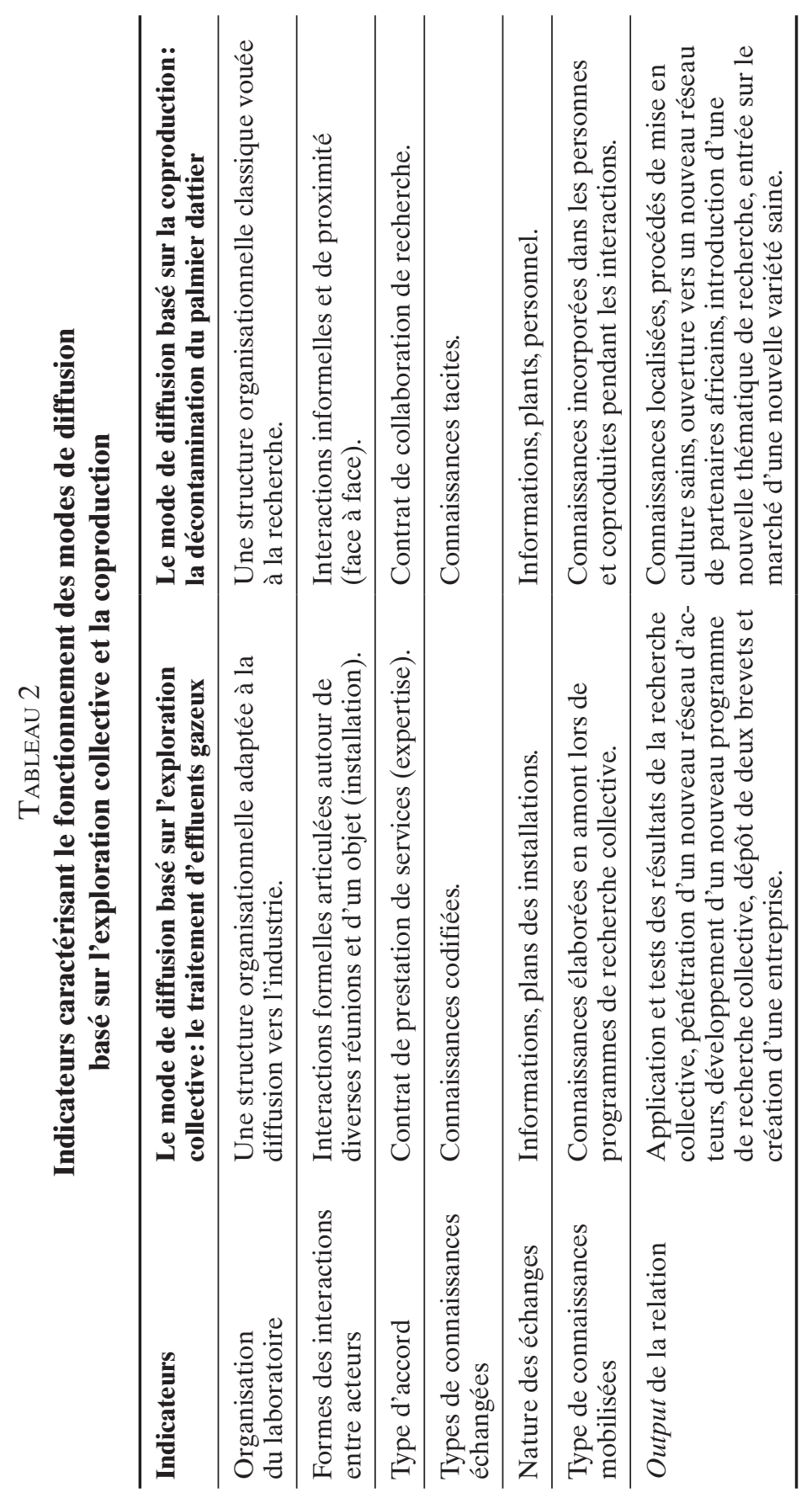

Revue internationale P.M.E., vol. 20, nº 1, 2007 
Ces deux illustrations mettent également en avant le rôle des procédures et routines propres à chaque laboratoire pour la gestion de la phase de contractualisation. Le GRETh n'a pas sollicité l'intervention de la fonction d'interface du CEA pour effectuer l'ensemble des démarches liées à la rédaction et la négociation des contrats. Pour ce faire, Monsieur « $\mathrm{M} »$, chargé d'affaires au GRETh, a utilisé les procédures et modèles mis à sa disposition par son laboratoire. Au contraire, le laboratoire INRA a fait appel à la fonction d'interface lors de la phase de contractualisation pour prendre en charge les problèmes de propriété industrielle et de rédaction. Au-delà de l'asymétrie des caractéristiques des deux modes, ces deux cas mettent en exergue les éléments clés, propres à chaque modèle de diffusion.

Le cas de «la décontamination des palmiers dattiers» est profondément marqué par la présence au sein du même laboratoire de membres issus de l'institut de recherche et de salariés de l'entreprise, caractéristique qui reste assez rare lors d'une coopération entre un laboratoire public et une PME. Dans le cadre d'une configuration de coproduction liant un laboratoire de recherche public et une PME, la présence d'une «personne-ressource» comme la jeune détentrice d'un doctorat de l'INRA, peut être perçue comme un élément clé de la réussite du processus de diffusion. Cette personne a un rôle de «traducteur» et «transmetteur» qui rend possible l'échange de connaissances tacites entre le laboratoire de recherche et la PME. Autrement dit, l'existence de ce «traducteur-transmetteur» permet au mode de diffusion basé sur la coproduction de fonctionner aussi avec des PME à faible intensité de recherche en surmontant le problème de capacité d'absorption. La présence d'une «personne-ressource» lors de la coopération permet donc au mode de coproduction de dépasser ses limites et de contribuer au transfert de connaissances entre la recherche publique et les PME traditionnelles. Ainsi, si ce modèle de diffusion ne semble pas le mieux adapté au transfert des connaissances vers les PME, il peut néanmoins atteindre cet objectif lorsqu'une "personne-ressource » joue le rôle du maillon manquant capable de fonctionner comme un «traducteur-transmetteur» entre le laboratoire de recherche public et la PME.

Dans le cas du traitement des effluents gazeux, les connaissances existaient ex-ante; elles ne résultent pas d'un processus de coproduction entre les partenaires participants. Leur production est le résultat de processus de recherche collective en amont. En effet, les connaissances utilisées par le GRETh lors de sa coopération avec la $\mathrm{PME}$ «S» sont le résultat de programmes de recherche déjà effectués. Toutefois, la prestation a largement contribué à l'enrichissement des connaissances fondamentales qui ont ainsi pu être appliquées et testées empiriquement. On comprend alors que pour fonctionner, le mode de diffusion basé sur l'exploration collective exige 
que le laboratoire impliqué soit fortement connecté en amont à un réseau de laboratoires partenaires plus fondamentaux et dispose de connaissances génériques stabilisées.

Le fonctionnement du mode de diffusion basé sur l'exploration collective tient à l'organisation du processus de recherche collectif. Dans le laboratoire du GRETh, ce processus est interne et ne dépend pas de l'intervention de structures externes. La diffusion des résultats de cette recherche est organisée; différents dispositifs ont été mis en place dans ce but. Il en est de même pour la mise en relation qui dépend d'une cellule et d'un club qui ont été créés à cet effet. On voit ainsi que le bon fonctionnement du mode de diffusion basé sur l'exploration collective dépend en grande partie de la structure organisationnelle du laboratoire impliqué.

L'importance de cette composante organisationnelle devient d'autant plus évidente lorsqu'on la met en perspective sur l'ensemble des 48 cas de coopération étudiés. En effet, lorsqu'on croise les modes de production/diffusion des connaissances mobilisés dans les 48 cas de coopération étudiés et la combinaison organisationnelle des 20 laboratoires impliqués, on remarque que seules les cinq unités de recherche dotées de dispositifs organisationnels adaptés ont pu adopter un modèle d'exploration collective ( $c f$. tableau 3 ).

Plusieurs dispositifs organisationnels comme une cellule de diffusion technologique interne, des plates-formes industrielles ou halles de technologies et une division du travail qui prend en compte l'activité de diffusion vers l'industrie sont nécessaires pour soutenir ce modèle. Par contre, la mobilisation du mode de diffusion basé sur la coproduction ne requiert pas une combinaison organisationnelle spécifique, tous les laboratoires de notre échantillon peuvent emprunter ce mode. Le profil de l'entreprise coopérante (dotation en R-D interne, intensité technologique, capacité d'absorption) semble beaucoup plus déterminant pour la mise en œuvre de ce mode. 


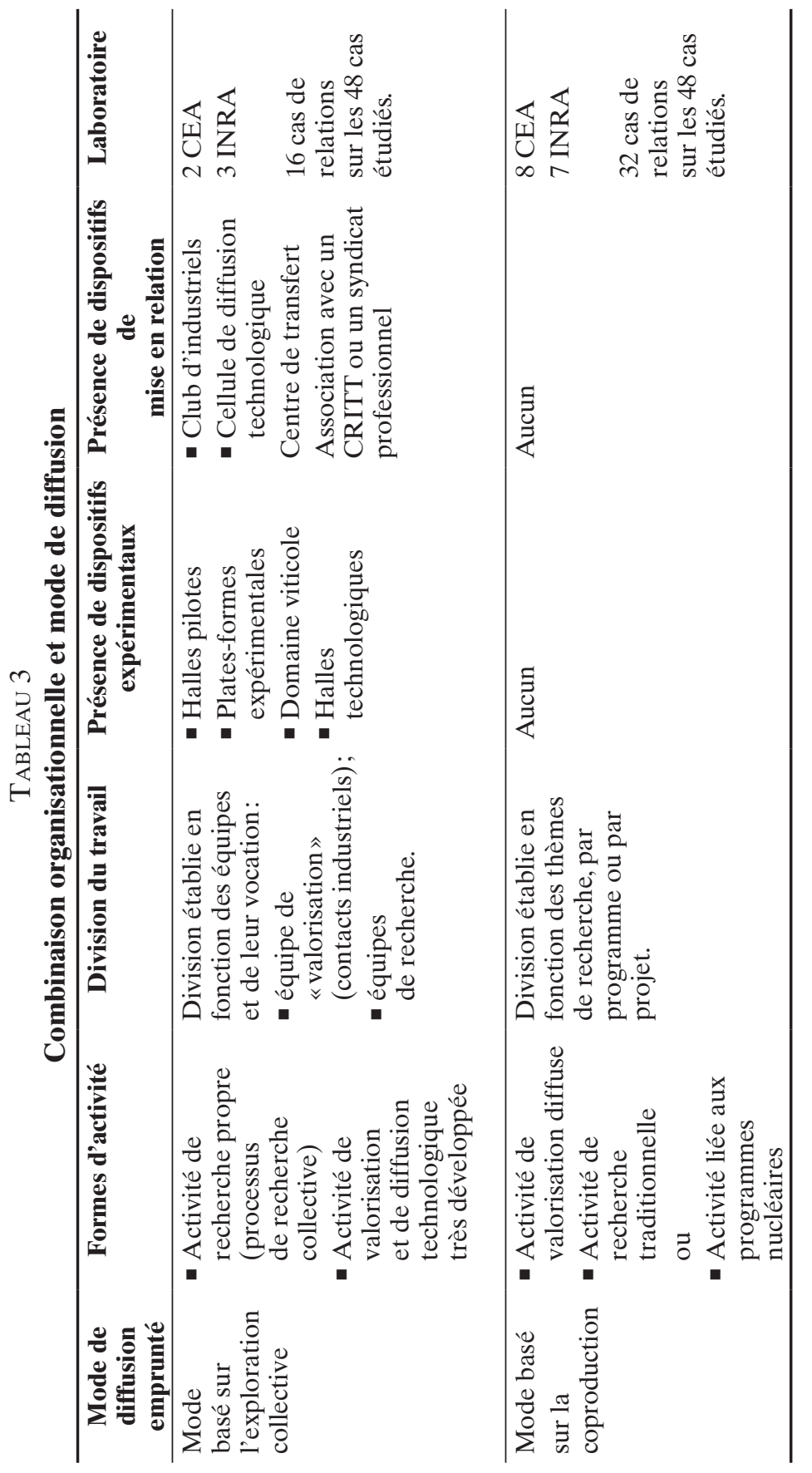

Revue internationale P.M.E., vol. 20, nº 1, 2007 


\section{Conclusion}

Notre analyse a montré que la diffusion des connaissances vers les PME peut prendre deux formes alternatives : un modèle basé sur la coproduction et un modèle basé sur l'exploration collective. Si le premier modèle se rapproche du cas généralement mis en avant par les économistes du changement technique, le second reste original, car il est le seul capable d'engendrer des connaissances standardisées et réutilisables. En ce sens, le modèle d'exploration collective est adapté aux PME faiblement intensives en recherche.

En effet, dans le mode de coproduction, pour que l'entreprise soit en mesure de coproduire et d'assimiler des connaissances, elle doit être dotée d'une forte capacité d'absorption. Or, nous avons vu que si les grandes entreprises et les PME high-tech sont souvent pourvues de départements de recherche internes et inscrivent à leur actif plusieurs expériences de collaboration, il n'en est pas de même pour les PME à plus faible capacité de recherche. De plus, le mode de coproduction exige qu'à chaque coopération l'entreprise participante réalise à nouveau de lourds investissements (création de nouvelles connaissances tacites, nouveau contexte d'application, acquisition de nouvelles connaissances scientifiques et techniques, etc.). De telles contraintes expliquent en partie pourquoi, dans un contexte de coproduction, les instituts de recherche publics éprouvent autant de difficultés à diffuser des connaissances vers les PME à faible intensité de recherche. Grâce aux différents dispositifs (le processus de recherche collective, les halles technologiques, les plates-formes industrielles, etc.) qui caractérisent le mode de diffusion basé sur l'exploration collective, le second modèle contourne ces difficultés. Toutefois, ce modèle reste encore assez peu mobilisé; en effet, sur les 48 coopérations analysées, seul un tiers des cas ont adopté le mode d'exploration collective. Ce résultat s'explique en partie par les spécificités organisationnelles à mettre en place pour le fonctionnement de ce modèle qui ne correspondent pas à la structure classique des laboratoires publics de recherche.

Pour dépasser le problème de la capacité d'absorption limitée des PME à faible intensité de recherche, les politiques publiques de soutien à l'innovation devraient donc davantage s'appuyer sur un mode de production/diffusion basé sur l'exploration collective. Une telle inflexion politique conduirait les organismes de recherche à mettre en place des laboratoires spécialisés dont l'objectif premier ne serait pas la production scientifique certifiée mais la production de connaissances standardisées et réutilisables. 


\section{Bibliographie}

Annales des Mines (1998), «Organisations de recherche», Réalités industrielles, février.

Acs, Z., D. Audretsch et M. Feldman (1992), «Real effects of academic research: comment», American Economic Review, vol. 82, p. 363-367.

Argyris, C. et D.A. SCHÖN (2002), Apprentissage organisationnel: théorie, méthode et pratique, Bruxelles, De Boeck Université.

Arora, A. et A. Gambardella (1994), «Evaluating technological information and utilising it», Journal of Economic Behavior and Organization, vol. 78, n ${ }^{\circ} 4$, p. 678-690.

Audretsch, D.B., B. Bozeman, K.L. Combs, M. Feldman, A.N. Link, D.S. Siegel, P. Stephan, G. TAssey et C. Wessner (2002), «The economics of science and technology », Journal of Technology Transfer, vol. 27, n 2, p. 155-203.

Audretsch, D.B. et M.P.FeldMAn (1996), «R-D spillovers and the geography of innovation and production », American Economic Review, vol. 86, n 3, p. 630-640.

Chabbal (1993), «L'innovation dans les PME», rapport de la Direction de l'innovation de la technologie et de l'action régionale et de la Direction de l'action régionale et de la moyenne et petite industrie, novembre.

CAllon, M. (1997), «Analysis of strategic relations between firms and university laboratories », Conference on the Need for a New Economics of Science, University of Notre-Dame.

Cohen, W.M. et D.A. Levinthal (1989), «Innovation and learning: the two faces of R-D», Economic Journal, vol. 99, p. 569.

Commission européenne (1995), Livre vert sur l'innovation, CECA-CE-CEA, Bruxelles, Luxembourg, 1995.

Guillaume, H. (1998), Rapport de mission sur la technologie et l'innovation, Paris, MENRT, Ministère de l'Économie et des Finances.

JAFFE, A. (1989), «Real effects of academic research», American Economic Review, vol. 79, p. 957-970.

Joly, P.B. et V. Mangematin (1996), «Profile of laboratories, industrial partnerships and organization of R-D: the dynamics of relations with industry in a large research organization», Research Policy, vol. 25, n 4, p. 901-922.

Julien, P.-A. (2004), «Actionnabilité de la recherche universitaire: du temps long au temps allongé. Le cas de la Chaire Bombardier», Revue internationale PME, vol. $17, \mathrm{n}^{\text {os }} 3-4$, p. 69-94.

Link, A.N. et J. ReEs (1990), «Firm size, university based research and the returns to R-D», Small Business Economics, vol. 2, n 1, p. 25-32.

Mangematin, V. (1997), «De l'assimilation des connaissances à la capacité de gestion. Les PMI de l'agro-alimentaire dans la région Rhône-Alpes », Les cahiers d'économie et de sociologie rurales, vol. 44, p. 83-105. 
Mangematin, V. et L. Nesta (1999), «What kind of knowledge can a firm absorb?»,

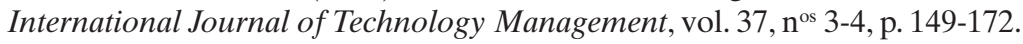

MANSFIELD, E. (1995), «Academic research underlying industrial innovations : sources, characteristics and financing», Review of Economics and Statistics, vol. 75, $\mathrm{n}^{\circ} 1$, p. 55-65.

ORgANISATION DE COOPÉRATION ET DE DÉVELOPPEMENT ÉCONOMIQUES - OCDE (2004), «Small and medium enterprises: seizing the potential», The OECD Observer, vol. 243, p. 46-48.

Pavitt, K., M. Robson et J. Townsend (1987), «The size distribution of innovating firms in the UK: 1945-1983», The Journal of Industrial Economics, vol. 55, no 3, p. 291-316.

Polanyi, M. (1962), Personal Knowledge: Towards a Post Critical Philosophy, Londres, Routledge, 428 pages.

Rosenberg, N. (1982), Inside the Black Box. Technology and Economics, Cambridge, Cambridge University Press.

RosenberG, N. (1990), «Why companies do basic research with their own money?», Research Policy, vol. 19, p. 165-174.

SAviotti (1994), Knowledge, Information, and Organizational Structures, Milan.

Siegel, D.S., J.G. Thursby, M.C. Thursby et A.A. Ziedonis (2001), «Organizational issues in university-industry technology transfer: an overview of the symposium issue », Journal of Technology Transfer, vol. 26, $\mathrm{n}^{\text {os }}$ 1-2, p. 5-11.

Senker, J. (1995), «Networks and tacit knowledge in innovation», Économies et Sociétés, vol. 9, n 2, p. 99-118.

SESSI - DiGITIP (2003-2004), L'industrie en quelques chiffres, Édition 2003-2004.

Revue internationale P.M.E., vol. 20, nº 1, 2007 